\title{
The Clinical Significance of miR-34a in Pancreatic Ductal Carcinoma and Associated Molecular and Cellular Mechanisms
}

\author{
Li-Min Long Jun-Kun Zhan Hai-Qin Wang Shuang Li Yi-Yin Chen \\ You-Shuo Liu
}

Department of Geriatrics, Institute of Aging and Geriatrics, The Second Xiangya Hospital, Central South University, Changsha, PR China

\section{Key Words}

Pancreatic duct cancer $\cdot \mathrm{miR}-34 \cdot \mathrm{miR}-150$.

Chemoresistance $\cdot$ Notch signaling

\begin{abstract}
Background: Pancreatic ductal adenocarcinoma (PDAC) exhibits poor prognosis and resistance to chemotherapy. This study was to identify the biomarkers associated with the progression, poor prognosis and chemoresistance of PDAC. Methods: miR-34a and miR-150 levels in the plasma and tissues from PDAC patients were measured by real-time PCR. Xenograft PDAC tumor models were established in mice by inoculation of CD133+ stem cells isolated from PDAC tumors. Protein expression was measured by Western blot. $R \boldsymbol{e}$ sults: The plasma miR-34a and miR-150 levels were significantly lower in PDAC patients than in patients with benign pancreatic lesions and in healthy subjects. The miR-34a and miR-150 levels in the tumor tissues were significantly lower than in pancreatic tissues with benign lesions. The protein levels of CD133, Notch1, Notch2 and Notch4 receptors in PDAC tumor tissues were significantly higher than in pancreatic tissues with benign lesions. miR-34a injection significantly inhibited the tumor growth of PDAC tumors and sensitized the anticancer effects of 5-fluorouracil (5-FU). miR-
\end{abstract}

34a significantly inhibited Notch1, Notch2 and Notch4 expression in xenograft tumor tissues in vivo and BxPC-3 cells in vitro. miR-34a and miR-150 significantly induced apoptosis and inhibited proliferation, invasion and migration in BxPC-3 cells. miR-34a, but not miR-150, significantly sensitized the anticancer effect of 5-FU in BxPC-3 cells in vitro. Conclusion: A loss of expression of miR-34a, but not of miR-150, is associated with disease progression and poor prognosis in PDAC patients, and may be involved in the chemoresistance of PDAC cells.

๑) 2016 S. Karger AG, Basel

\section{Introduction}

Pancreatic ductal adenocarcinoma (PDAC) is a highly aggressive tumor of the pancreas and the fourth leading cause of cancer-related deaths worldwide [1]. A report from the National Cancer Data Base estimated that there were 39,590 pancreatic cancer deaths in the USA in 2014 [2]. Despite the progress made in diagnostic and therapeutic technology, the 5 -year survival rate is only $23 \%$ in patients with local disease [3] and $<1 \%$ in patients with unresectable tumors [4]. The low survival rate might be associated with the aggressive characteristics of PDAC

You-Shuo Liu, MD, $\mathrm{PhD}$

Department of Geriatrics, The Second Xiangya Hospital, Central South University 139 Middle Renmin Road

Changsha, Hunan 410011 (PR China)

E-Mail karger@karger.co

E-Mail Liuyoushuo@yeah.net 
and the lack of biomarkers that can facilitate an early diagnosis and target therapy of pancreatic cancer.

MicroRNAs (miRNAs) are a family of small, noncoding, endogenously expressed RNA molecules, made up of 18-25 nucleotides. miRNAs can regulate gene expression at the transcriptional level by posttranscriptionally degrading the target mRNAs or just repressing their translation through binding to the 3 prime untranslated region (3'-UTR) [5]. The regulatory roles of miRNAs have been widely observed to participate in tumorigenesis, tumor cell differentiation, proliferation, apoptosis and metastasis of various cancers [6]. However, most of the functional roles and targets of miRNAs have not been identified. Reduced expression of miR-34a is associated with poor overall survival in PDAC patients following resection [7] and serum miR-34a levels are potential diagnostic biomarkers of PDAC [8]. Exogenous overexpression of miR$34 \mathrm{a}$ inhibits the proliferation of cultured pancreatic cancer cells [9], sensitizes MiaPaCa2 pancreatic cancer cells to chemo- and radiotherapy by downregulating $\mathrm{Bcl}-2$, Notch1 and Notch2 expression [10]. It also inhibits cell growth and induces apoptosis in pancreatic cancer cells by directly targeting and inhibiting Notch1 signaling [11]. miR-150 has been demonstrated to target Notch3 of the Notch receptor family and plays an important role in T-cell differentiation and leukemogenesis [12]. miR-150 overexpression can inhibit the malignant behavior of pancreatic cancer [13]. However, the clinical significance of miR-150 in PDAC has not been identified.

In this study, we measured the circulating miR-34a and miR-150 levels in PDAC patients compared with healthy subjects and the expression of these miRNAs in tumor tissues compared with benign pancreatic lesions. We further investigated the regulative effects of miR-34a on the Notch receptors in vitro and in vivo, through its overexpression.

\section{Materials and Methods}

\section{Clinical Data}

The serum and tumor tissues of 159 patients with PDAC tumors, serum and pancreatic tissues of 82 patients with benign pancreatic lesions and serum of 44 age- and gender-matched healthy subjects were collected from January 2006 to December 2011 prior to radiotherapy or chemotherapy. Sixty-seven of the 159 patients (42.1\%) with PDAC were female and 92 (57.9\%) were male. Average age was $54.10 \pm 11.03$ years. Among the 159 PDAC tumors, 58 (36.5\%) were well differentiated, 52 (32.7\%) were moderately differentiated and 49 (30.8\%) were poorly differentiated adenocarcinomas. Maximum tumor size was $<3 \mathrm{~cm}$ in 19 (12.0\%) patients, $3-5 \mathrm{~cm}$ in $105(66.0 \%)$ patients and $>5 \mathrm{~cm}$ in 35 (22.0\%) patients.
Of the 159 patients with PDAC, 44 (27.7\%) had regional lymph node metastasis while 115 had no lymph node metastasis $(72.3 \%)$, and $96(60.4 \%)$ had invasion of the surrounding tissues and organs of the pancreas and $63(39.6 \%)$ had no invasion. Sixteen $(10.1 \%)$, $63(39.6 \%), 55(34.6 \%)$ and $25(15.7 \%)$ patients had TNM clinical stage I, II, III and IV disease, respectively. The survival information of all patients was collected by letters or telephone calls, with a follow-up period of 2 years. One hundred and nineteen patients survived for $<1$ year, 36 survived for $>1$ year and 4 survived for $>2$ years (censored cases). The average survival time was $9.62 \pm 0.45$ months.

Eighty-two patients with benign pancreatic lesions included 44 (53.7\%) males and 38 (46.3\%) females. Nineteen $(23.2 \%)$ with benign lesions were $\leq 45$ years old and $63(76.8 \%)$ were $>45$ years old. The pathological examination identified chronic pancreatitis in 30 (36.6\%) patients, adenoma in $30(36.6 \%)$ and intraepithelial neoplasia in $22(26.8 \%)$.

\section{Real-Time PCR}

Ten milliliters of peripheral fasting blood was collected. The plasma was harvested by centrifugation at 1,500 rpm for $5 \mathrm{~min}$ and frozen at $-80^{\circ} \mathrm{C}$. Tumor tissues were snap-frozen. Total RNA was isolated from plasma, tumor tissues and pancreatic tissues with benign lesions using TRIzol reagent (Invitrogen, Carlsbad, Calif., USA) by following the manufacturer's instructions. Five micrograms of total RNA was used for the reverse-transcription reaction using a One Step PrimeScript ${ }^{\circledR}$ miRNA cDNA synthesis kit by following the manufacturer's instructions. Real-time quantitative PCR of miR-34a and miR-150 was performed using SYBR ${ }^{\circledR}$ Premix Ex Taq ${ }^{\mathrm{TM}}$ II (TaKaRa, Japan). The sequences of mature miR$34 \mathrm{a}$ and $\mathrm{miR}-150$ were used to synthesize the forward primers. The reverse primers were provided by the kit.

\section{CD133+ Cell Isolation}

The tumor tissues were homogenized immediately after surgery and then trypsinized. Tumor cells from 5 PDAC patients were mixed. The mixed tumor cells were suspended at a concentration of $1 \times 10^{6} / \mathrm{ml}$ in $37^{\circ} \mathrm{C} \mathrm{DMEM} / \mathrm{F} 12 \mathrm{~K}$ medium. To sort CD133+ cells, each $10^{6}$ cells were incubated with FITC-anti-human CD133 monoclonal antibody (BioSS, China) for $1 \mathrm{~h}$ in the dark, rinsed with $1 \times$ PBS and then treated with permeabilization solutions. CD133+ cells were sorted with MoFlo flow cytometry (Dako, Carpinteria, Calif., USA). CD133+ subpopulations from PDAC tumor tissues were directly used for inoculation in mice.

\section{Tumor Growth}

Eighty BALB/C nude mice (BALB/c, nu/nu) weighing 20-21 g were purchased from Shanghai Biological Science Institution and housed according to the Animal Care Guidelines of the Chinese Council. The animal protocol was approved by the Animal Use Committee of Central South University. Approximately $1 \times 10^{6}$ CD133+ PDAC tumor cells were subcutaneously injected into the right hind limbs of the mice. After the tumors grew to 7-8 $\mathrm{mm}$ in size, the mice were randomly divided into 4 groups $(n=20$ in each). The control group received control siRNA injection ( $5^{\prime}$-AAUUCUCCGAACGUGUCACGU-3'), the 5-fluorouracil (5-FU) group received i.p. injection, the $5-\mathrm{FU}+$ siR-34a group received miR-34a mimic (5'-UGGCAGUGUCUUAGCUGGUUGU- $3^{\prime}$ ) and 5-FU injection and the miR-34a alone group received miR-35a mimic injection. 5-FU was injected intraperitoneally at a 
Table 1. Associations of miR-34a and miR-150 protein expression and clinicopathological characteristics of PDAC

\begin{tabular}{|c|c|c|c|c|c|c|c|}
\hline \multirow[t]{2}{*}{ Characteristic } & \multirow[t]{2}{*}{ Cases, $\mathrm{n}$} & \multicolumn{3}{|c|}{ miR-34a high } & \multicolumn{3}{|c|}{ miR-150 high } \\
\hline & & $\mathrm{n}(\%)$ & $\chi^{2}$ & $\mathrm{p}$ value & $\mathrm{n}(\%)$ & $x^{2}$ & $\mathrm{p}$ value \\
\hline \multicolumn{8}{|l|}{ Age } \\
\hline$\leq 45$ years & 33 & $17(51.5)$ & \multirow[t]{2}{*}{0.000} & \multirow[t]{2}{*}{0.994} & $16(48.9)$ & \multirow[t]{2}{*}{0.015} & \multirow[t]{2}{*}{0.903} \\
\hline$>45$ years & 126 & $65(51.6)$ & & & $65(51.6)$ & & \\
\hline \multicolumn{8}{|l|}{ Sex } \\
\hline Male & 92 & $51(55.4)$ & \multirow[t]{2}{*}{0.963} & \multirow[t]{2}{*}{0.326} & $43(46.7)$ & \multirow[t]{2}{*}{1.171} & \multirow[t]{2}{*}{0.279} \\
\hline Female & 67 & $31(46.3)$ & & & $38(56.7)$ & & \\
\hline \multicolumn{8}{|l|}{ Tumor } \\
\hline Well-differentiated & 58 & $31(53.5)$ & \multirow[t]{3}{*}{2.35} & \multirow[t]{3}{*}{0.309} & $28(48.3)$ & \multirow[t]{3}{*}{0.34} & \multirow[t]{3}{*}{0.843} \\
\hline Moderately differentiated & d 52 & $30(67.7)$ & & & $28(53.8)$ & & \\
\hline Poorly differentiated & 49 & $21(42.9)$ & & & $25(51.0)$ & & \\
\hline \multicolumn{8}{|l|}{ Tumor size } \\
\hline$\leq 3 \mathrm{~cm}$ & 19 & $12(63.2)$ & \multirow[t]{3}{*}{4.25} & \multirow[t]{3}{*}{0.120} & $9(47.4)$ & \multirow[t]{3}{*}{0.11} & \multirow[t]{3}{*}{0.946} \\
\hline $3-5 \mathrm{~cm}$ & 105 & $57(54.3)$ & & & $54(51.4)$ & & \\
\hline$>5 \mathrm{~cm}$ & 35 & $13(37.1)$ & & & $18(51.4)$ & & \\
\hline \multicolumn{8}{|l|}{ Lymph node metastasis } \\
\hline No & 115 & $70(60.9)$ & \multirow[t]{2}{*}{13.069} & \multirow[t]{2}{*}{$<0.001$} & $56(48.7)$ & \multirow[t]{2}{*}{0.547} & \multirow[t]{2}{*}{0.460} \\
\hline Yes & 44 & $12(27.3)$ & & & $25(56.8)$ & & \\
\hline \multicolumn{8}{|l|}{ Invasion } \\
\hline No & 63 & $52(82.5)$ & \multirow[t]{2}{*}{38.038} & \multirow[t]{2}{*}{$<0.001$} & $31(49.2)$ & 0.037 & 0.847 \\
\hline Yes & 96 & $30(20.8)$ & & & $50(52.1)$ & & \\
\hline TNM stage & & & & & & & \\
\hline $\mathrm{T} 1$ & 16 & $14(87.5)$ & 34.87 & $<0.001$ & $12(75.0)$ & 11.34 & $0.011^{*}$ \\
\hline $\mathrm{T} 2$ & 63 & $43(68.3)$ & & & $38(60.3)$ & & \\
\hline $\mathrm{T} 3$ & 55 & $21(38.2)$ & & & $23(41.8)$ & & \\
\hline $\mathrm{T} 4$ & 25 & $3(12.0)$ & & & $8(32.0)$ & & \\
\hline
\end{tabular}

$* \mathrm{p}<0.05$.

dose of $50 \mathrm{mg} / \mathrm{kg} 3 \times$ daily for 4 days (i.p.). Ten micrograms of miR34 a was intratumorally injected $2 \times$ per week for 4 weeks. Animals were killed 30 days after treatment. Tumor volume was calculated with the formula: $\left(\mathrm{L} \times \mathrm{W}^{2}\right) / 2$, where $\mathrm{L}$ is the tumor length and $\mathrm{W}$ is the tumor width. Both were measured with a caliper. The mice were sacrificed at day 30 and the tumor tissues were excised.

\section{Western Blot}

The tumor tissues were homogenized in ice-cold homogenization buffer containing protein and phosphatase inhibitors. Western blot was performed as previously described [14]. Antibodies for CD133 and Notch1-4 were purchased from Santa Cruz Biotechnology (Santa Cruz, Calif., USA). To control for loading efficiency, the blots were stripped and reprobed with $\alpha$-tubulin antibody (Abcam, Cambridge, Mass., USA). Proteins were normalized to $a$-tubulin and then expressed as a percentage of the control.

\section{Cell Culture and Treatments}

BxPC-3, a human pancreatic adenocarcinoma cell line, was originally purchased from American Type Culture Collection. BxPC- 3 cells were incubated in RPMI $1640+2 \mathrm{mM}$ glutamine medium. The complete medium contained $10 \%$ fetal bovine serum and $1 \%$ penicillin/streptomycin. Cells were cultured at $37^{\circ} \mathrm{C}, 5 \%$ $\mathrm{CO}_{2}$. BxPC-3 cells were seeded $24 \mathrm{~h}$ before treatments. Cells were transfected with miR-34a mimic (5'-UGGCAGUGUCUUAGCUGGUUGU-3 ${ }^{\prime}$ ), miR-150 mimic (5'-UCUCCCAACCCUUGUACCAGUG- $3^{\prime}$ ) and control miRNA ( $5^{\prime}$-AAUUCUCCGAACGUGUCACGU-3'), using Lipfectin-2000 (Invitrogen) according to the user manual. Six hours later, the Opti-MEM medium was completely replaced with RPMI 1640 medium and cells were continually cultured for $24 \mathrm{~h}$, followed by incubation with or without $0.1 \mathrm{mM}$ of 5 -FU as previously reported [15].

\section{Cell Viability Assay}

BxPC-3 cells were treated with control siRNA transfection, miR-34a mimic or miR-150 mimic transfection, 5-FU and 5-FU + miR-34a mimic or miR-150 mimic transfection as described above. Twenty-four hours later, cells were incubated with 3-(4,5-dimethylthiazol-2-yl)-2,5-diphenyltetrazolium bromide (MTT) dye according to a published protocol [16]. The cell viability was calculated as the percentage of absorbance of the samples compared to the controls.

\section{Apoptosis Assay}

Cells were treated as described above and cell death was visualized by Hoechst 33342 staining (Calbiochem, San Diego, Calif., USA) as previously described [16]. After treatment for $48 \mathrm{~h}$, cells were fixed in methanol/acetic acid (3:1) for $10 \mathrm{~min}$ at $4^{\circ} \mathrm{C}$, followed
40 


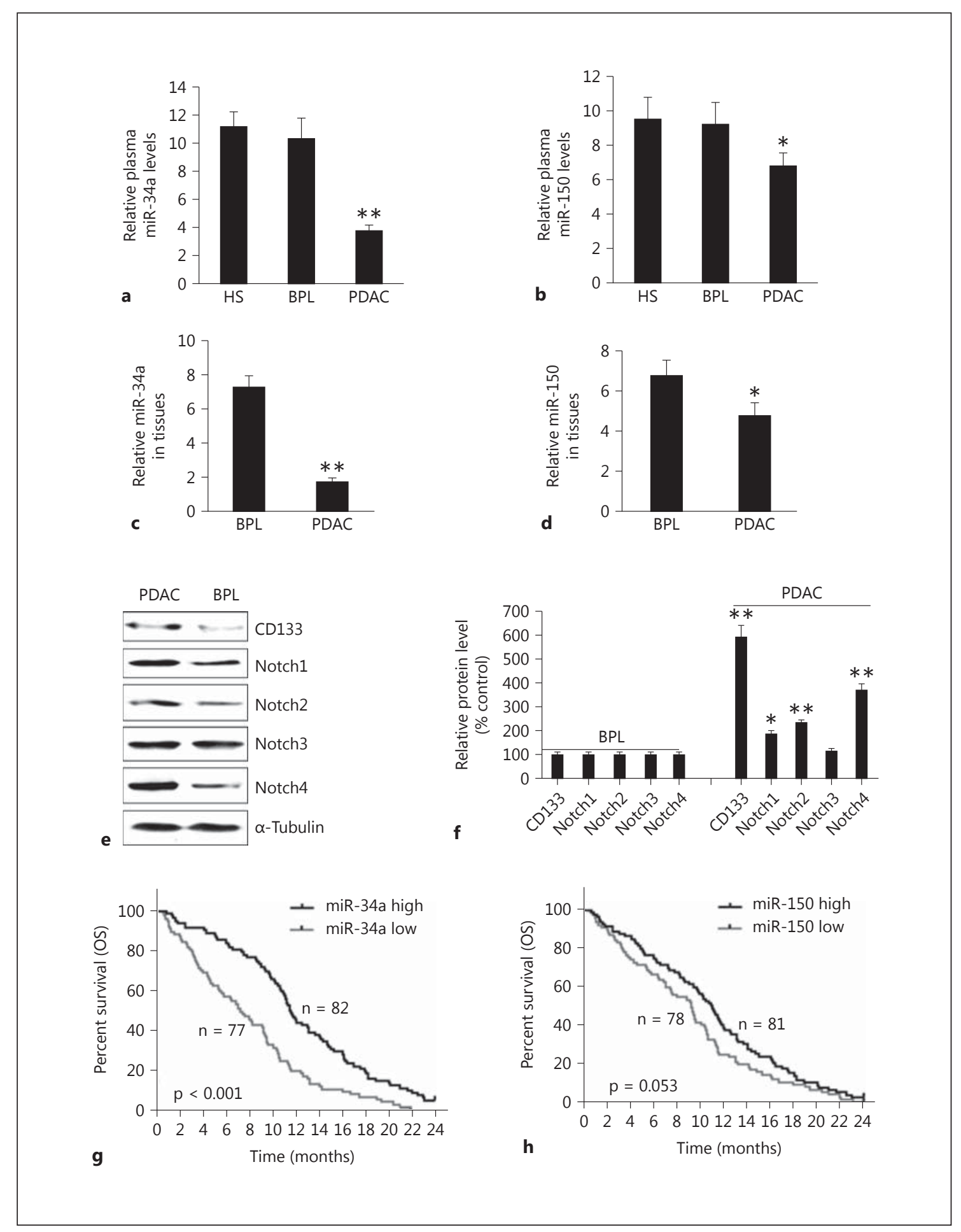

Fig. 1. miRNA and protein expression and the association of miRNAs with survival. a Plasma miR-34a levels in 44 healthy subjects (HS), 82 patients with benign pancreatic lesions (BPL) and 159 PDAC patients (PDAC). ${ }^{* *} \mathrm{p}<0.001$ versus other groups. $\mathbf{b}$ Plasma miR-150 levels. ${ }^{*} \mathrm{p}<0.05$ versus other groups. c miR-34a levels in PDAC tumor tissues and BPL. ${ }^{* *} \mathrm{p}<0.001$ versus BPL. d miR-150 levels in PDAC tumor tissues and BPL. ${ }^{*} \mathrm{p}<0.05$ ver- sus BPL. e Representative Western blots of protein expression in PDAC tumor tissues and BPL. $\mathbf{f}$ Semiquantitative analysis of protein expression in e. Data are presented by a relative level to BPL (100\%). g Overall survival (OS) of PDAC patients with high and low miR-34a levels. $\mathbf{h}$ OS of PDAC patients with high and low miR-150 levels. 
by incubation with Hoechst $33342(5 \mu \mathrm{g} / \mathrm{ml})$ for $10 \mathrm{~min}$ at room temperature. Cell nuclei were observed under a fluorescence microscope with a DAPI filter. Apoptotic cells showed clear condensation and small bright nuclei. Cells in 5 random areas were counted. The average percentage of apoptotic nuclei was calculated.

Migration and Invasion Assays

To perform cell invasion and migration assays, cells were cultured in Matrigel-coated or noncoated 24-well Transwell plates (8- $\mu \mathrm{m}$ pore size, BD Biosciences, Franklin Lakes, N.J., USA), respectively. Briefly, BxPC-3 cells were transfected with or without miR-34a or miR-150 mimic for $24 \mathrm{~h}$. Cells were then harvested and suspended in RPMI 1640 medium containing $1 \%$ FCS. $1 \times 10^{5}$ cells were seeded into the upper chamber of each well and RPMI 1640 containing 20\% FCS was placed in the lower chamber. After $48 \mathrm{~h}$ of incubation, the membranes were fixed with methanol and stained with $1 \%$ toluidine blue in $1 \%$ borax. The cells on the membrane were counted under a light microscope.

\section{Statistics}

Data were presented as mean \pm standard error (SEM) and analyzed using SPSS v16.0 (Chicago, Ill., USA). The relationships between serum and tumor miR-34a and miR-150 levels and histological or clinical factors were analyzed by $\chi^{2}$ test or Fisher's exact test. The Kaplan-Meier method (log-rank test) was used for the univariate analysis of overall survival in patients with high or low levels of miR-34a or miR-150, i.e. levels higher or lower than the mean level for the 159 PDAC patients. One-way ANOVA or 2 -tailed Student's t test was used for comparison between groups. $\mathrm{p}<0.05$ was considered statistically significant.

\section{Results}

\section{The Clinical Significance of $m i R-34 a$ and $m i R-150$} Expression

The plasma miR-34a (fig. 1a) and miR-150 (fig. 1b) levels were significantly lower in PDAC patients than in the healthy subjects and the patients with benign pancreatic lesions ( $\mathrm{p}<0.001$ and $\mathrm{p}<0.05$, respectively). The miR-34a (fig. 1c) and miR-150 (fig. 1d) levels were significantly lower in PDAC tumors than in benign pancreatic lesions ( $p<0.001$ and $p<0.05$, respectively). The protein levels of CD133, Notch1, Notch2 and Notch4, but not Notch3, were significantly higher in PDAC tumor tissues than in benign pancreatic lesions $(\mathrm{p}<0.001$; fig. 1e, f). However, a high miR-34a level significantly correlated with larger tumor size $(\mathrm{p}<0.05)$, high TNM stage $(\mathrm{p}<0.01)$, invasion $(\mathrm{p}<0.001)$ and lymph node metastasis $(\mathrm{p}<0.001$; table 1$)$. A high miR-150 level significantly correlated only with high TNM stage ( $\mathrm{p}<$ $0.05)$. The univariate analysis showed that a low level of miR-34a (fig. 1g; $\mathrm{p}<0.001$ ), but not of miR-150 (fig. 1h; $\mathrm{p}=0.053)$, significantly correlated with shorter overall survival.

\section{miR-34a Inhibited Tumor Growth through Notch}

Signaling

To investigate the targeted signaling pathway of miR34a in PDAC tumors, the CD133+ cells were isolated from human PDAC tumors and inoculated into nude mice. After the tumors grew to about $7-8 \mathrm{~mm}$ in size, the mice were grouped and treated with or without synthesized miR-34a mimic, 5-FU or miR-34a mimic + 5-FU. Both miR-34a mimic and 5-FU significantly inhibited tumor growth from day $10(\mathrm{p}<0.05)$ to day $30(\mathrm{p}<0.001)$ after treatment. The combination of miR-34a mimic and 5 -FU injection was more effective in inhibiting tumor growth ( $\mathrm{p}<0.01$ from day 10; fig. $2 \mathrm{a})$. Real-time PCR validated a high level of miR-34a mimic in the tumor tissues injected with miR-34a mimic $(\mathrm{p}<0.001), 5$-FU injection alone $(\mathrm{p}<0.05)$ and miR-34a mimic $+5-\mathrm{FU}(\mathrm{p}<$ 0.001 ; fig. 2b). Western blot showed that injection of synthesized miR-34a mimic and miR-34a mimic + 5-FU significantly lowered the levels of CD133 (fig. 2c, d; p < 0.001), Notch1 (fig. 2c, e; p < 0.001), Notch2 (fig. 2c, f; p $<0.001$ ) and Notch4 (fig. 2c, h; p < 0.001), but not Notch3 (fig. 2c, g) protein. 5-FU injection alone significantly decreased the expression of CD133 (fig. $2 c, d ; p<0.05$ ), Notch1 (fig. 2c, e; p < 0.05) and Notch4 (fig. 2c, h; p < 0.05 ), but not Notch2 (fig. 2c, f; p > 0.05) and Notch3 (fig. 2c, g; $p>0.05$ ) protein.

\section{miR-34a Inhibited Tumor Cell Survival, Invasion and} Migration and Increased Cell Apoptosis

To further investigate the role of miR-34a in tumor cell biology, human pancreatic adenocarcinoma BxPC-3 cells were transfected with control miRNA and miR-34a mimic, incubated with 5-FU and treated with miR-34a mimic plus 5-FU. Results showed that miR-34a mimic, 5-FU treatment and the combination of miR-34a mimic and 5-FU treatment significantly reduced cell viability (fig. 3a; $\mathrm{p}<0.001$ ), reduced cell invasion (fig. $3 \mathrm{~b} ; \mathrm{p}<0.01$, $\mathrm{p}<$ 0.001 ) and inhibited cell migration (fig. $3 c ; p<0.001$ ). But the effect of the combination of miR-34a transfection and 5-FU treatment was more significant than miR-34a mimic transfection or 5-FU treatment alone. In contrast, miR34a mimic transfection, 5-FU treatment and the combination of miR-34a mimic transfection and 5-FU treatment significantly increased cell apoptosis in BxPC-3 cells (fig. $3 \mathrm{~d}$, e; $\mathrm{p}<0.001$ ). The combination was more effective than miR-34a mimic transfection or 5-FU treatment alone.
Long/Zhan/Wang/Li/Chen/Liu 

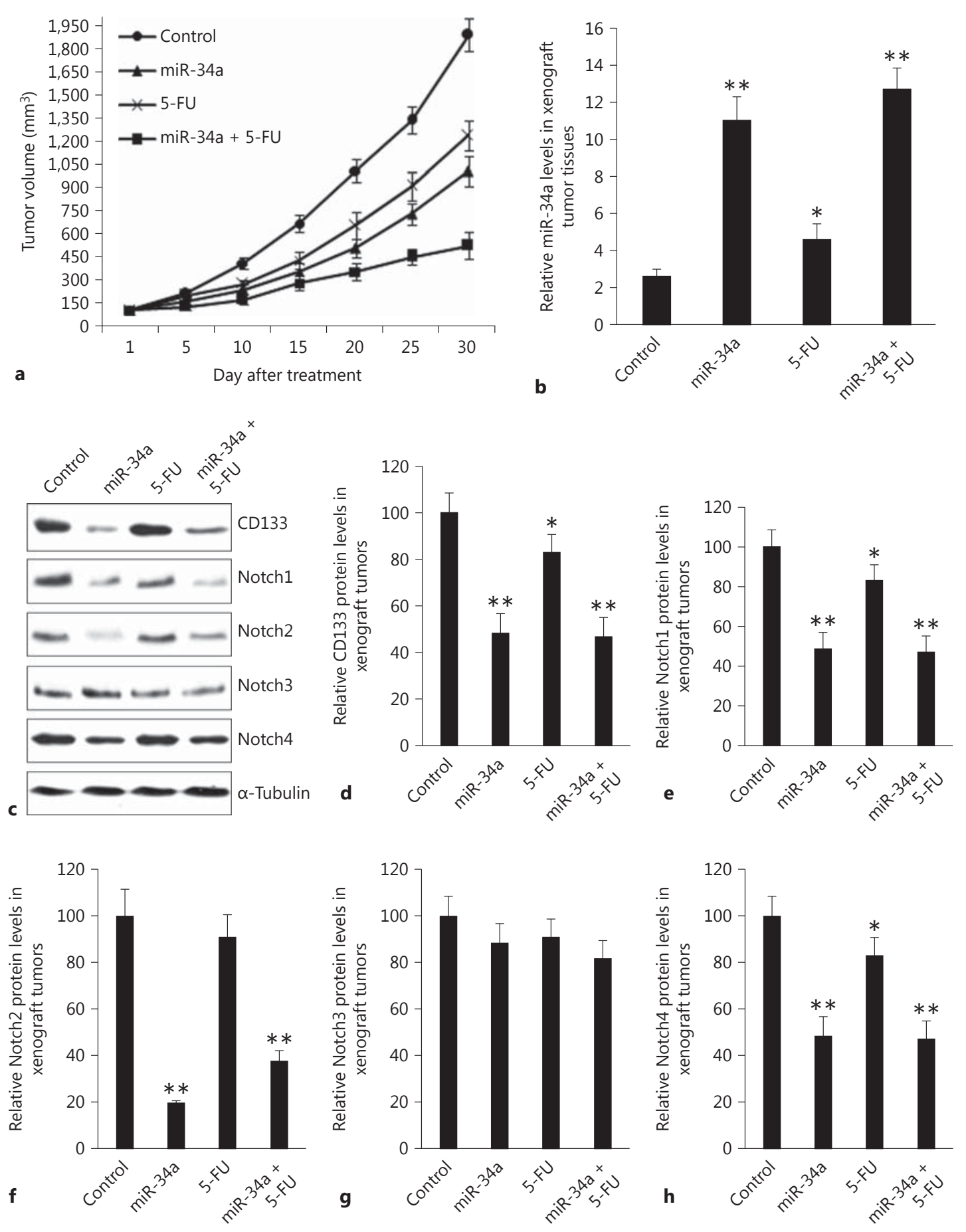

Fig. 2. Xenograft PDAC tumor growth and miR-34a, CD133 and Notch protein expression in tumor tissues. a Tumor growth after treatment. Mice were treated with intratumoral injection of control miRNA (control), miR-34a mimic (miR-34a), intraperitoneal injection of 5-FU (5-FU) and a combination of intratumoral miR$34 \mathrm{a}$ and intraperitoneal 5-FU injection (miR-34a +5-FU). b Rela- tive miR-34a levels in xenograft tumor tissues. ${ }^{*} \mathrm{p}<0.05$, ${ }^{* *} \mathrm{p}<$ 0.001 versus controls. c Representative Western blots of CD133, Notch1-4 and $\alpha$-tubulin protein. The bands in the Western blots were scanned and analyzed. $\mathbf{d}-\mathbf{h}$ The semiquantitative data for CD133 (d), Notch1 (e), Notch2 (f), Notch3 (g) and Notch4 (h) receptors are presented by a relative level to controls (100\%). 


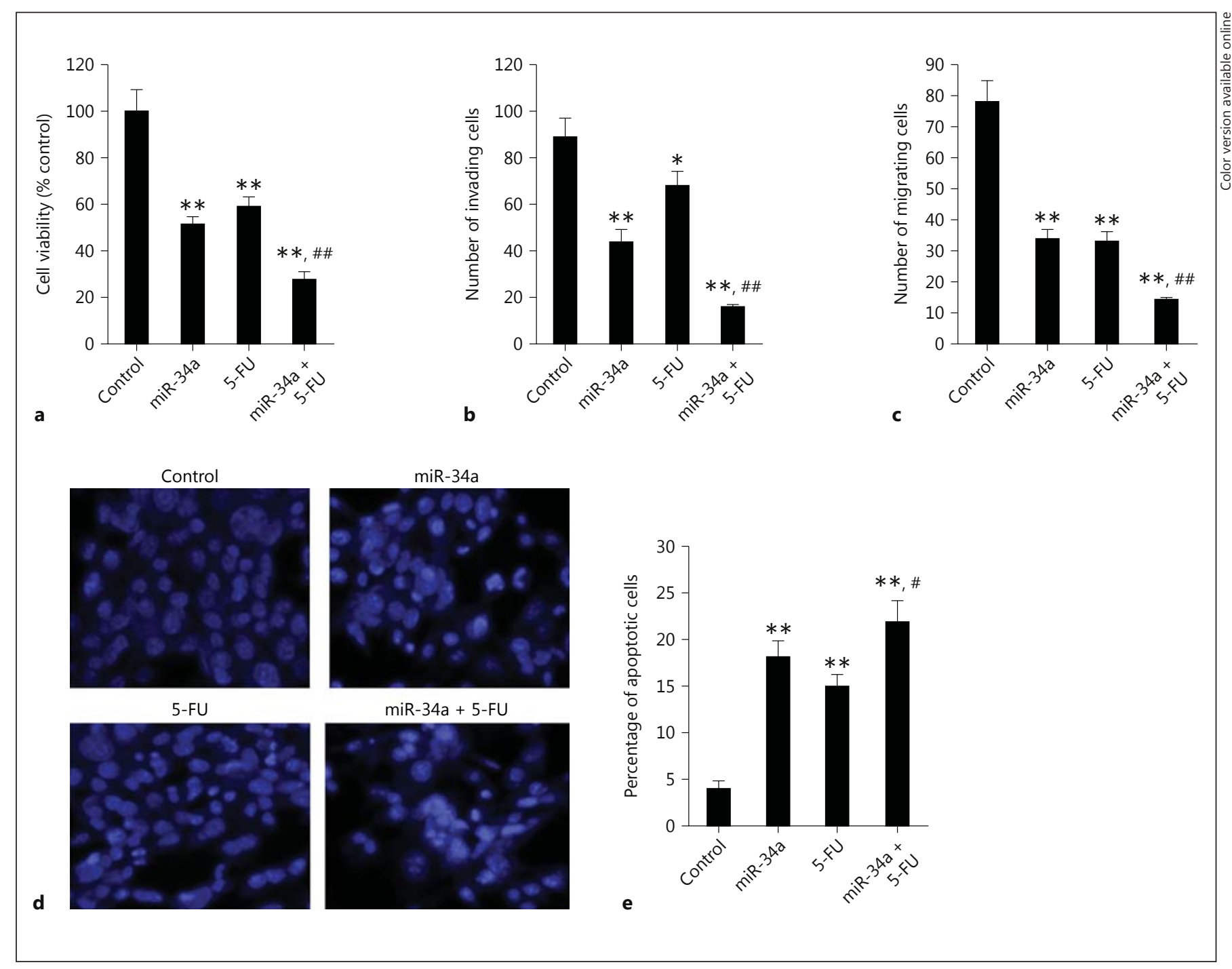

Fig. 3. The antitumor effect of miR-34a in BxPC-3 cells. Cells were transfected with miR-34a mimic, 5-FU incubation or combined miR-34a mimic transfection and 5-FU incubation. a Cell viability was analyzed by MTT. b Cell invasion analysis. c Cell migration analysis. d Representative Hoechst 33342 staining of treated BxPC3 cells. e Percentage of apoptotic cells on Hoechst 33342 staining. ${ }^{*} \mathrm{p}<0.05$, ${ }^{* *} \mathrm{p}<0.001$ versus controls. \# $\mathrm{p}<0.05$, \#\# $\mathrm{p}<0.001$ versus 5-FU treatment alone. $\mathrm{n}=5$.
miR-34a Mimic Inhibited Notch Signaling in BxPC-3 Cells

We further validated whether miR-34a also regulated Notch signaling in pancreatic tumor cells. BxPC-3 cells treated with control miRNA transfection, miR-34a mimic transfection, 5-FU or the combination of miR$34 \mathrm{a}$ mimic transfection and 5-FU, were subjected to Western blot analysis of Notch protein expression. miR34a mimic transfection significantly downregulated CD133 (fig. 4a, b; p < 0.001), Notch1 (fig. 4a, c; p < 0.001 ), Notch2 (fig. 4a, d; p < 0.001) and Notch4 (fig. 4a, f; $p<0.001$ ), but not Notch3 (fig. 4a, e; p > 0.05) protein expression. In contrast, 5-FU showed no effects on the expression of CD133 and Notch1-4 receptors (fig. 4; $\mathrm{p}>0.05)$.

miR-150 Inhibited Tumor Cell Survival, Invasion and Migration and Increased Cell Apoptosis Not through

Notch3 Signaling

To investigate whether miR-150 regulates Notch 3 expression and subsequently changes the phenotype of PDAC tumor cells, BxPC-3 cells were transfected with 


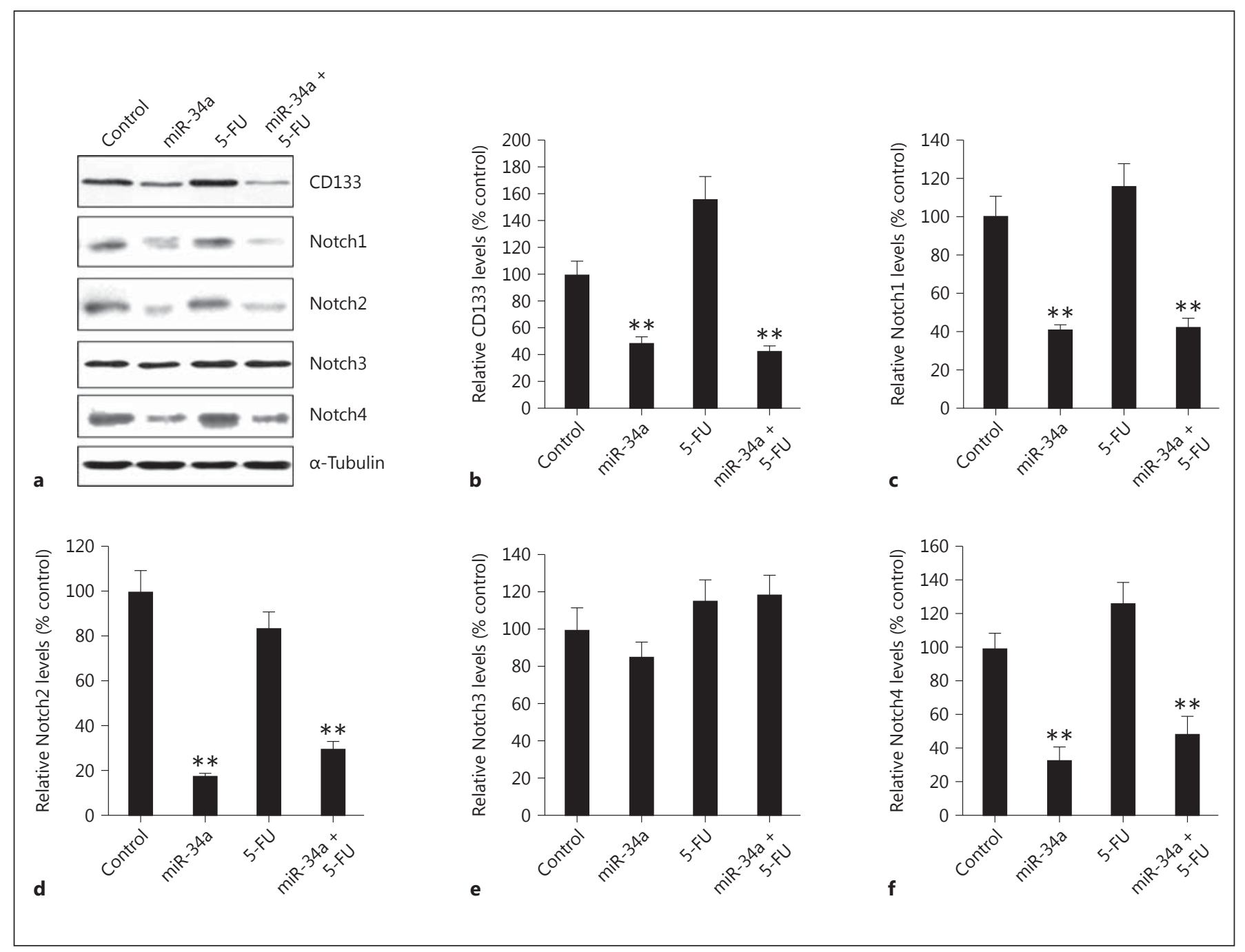

Fig. 4. Western blot analysis of protein expression in BxPC-3 cells. a Representative Western blots of CD133, Notch1-4 and $\mathbf{a}$-tubulin protein. b-f Semiquantitative analysis of CD133 (b), Notch1 (c), Notch2 (d), Notch3 (e) and Notch4 (f) receptor protein expression relative to controls $(100 \%)$. ${ }^{* *} \mathrm{p}<0.001$ vs. controls. $\mathrm{n}=5$.

or without miR-150 mimic, incubated with 5-FU and treated with miR-150 mimic + 5-FU. Results showed that both miR-150 transfection and 5-FU exhibited no effect on Notch3 protein expression (fig. $5 \mathrm{a}, \mathrm{b}$ ). miR-150 mimic, 5-FU treatment and the combination of miR150 mimic and 5-FU treatment significantly reduced cell viability (fig. 5c), increased cell apoptosis (fig. 5d), reduced cell invasion (fig. 5e), and inhibited cell migration (fig. 5f) ( $p<0.01$ or $p<0.001)$. But the effect of the combination of miR-150 transfection and 5-FU treatment was no more significant than 5 -FU treatment alone $(\mathrm{p}>0.05)$.

miR-34a in PDAC and Associated Molecular and Cellular Mechanisms

\section{Discussion}

miR-34a has been identified as a tumor suppressor in several tumors [17], but its clinical significance and associated molecular mechanisms have not been well documented in PDAC. A previous study demonstrated that forced expression of miR-150 can inhibit the malignant behavior of pancreatic cancer [13]. However, the clinical significance of miR-150 in PDAC patients has not been reported. Particularly, the roles that miR-34a and miR150 play in the drug resistance of PDAC tumor cells have not been comparably studied. This study demonstrated that miR-34a and miR-150 levels were significantly low- 

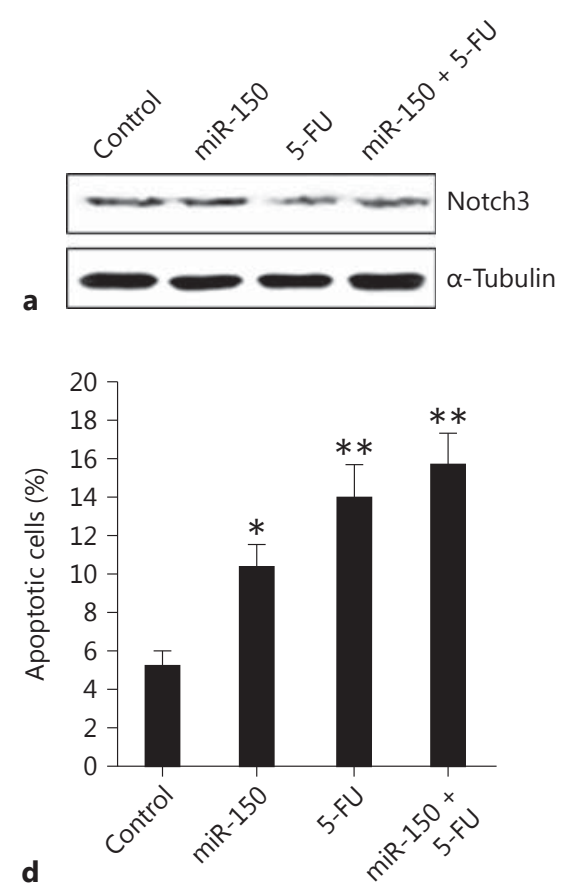
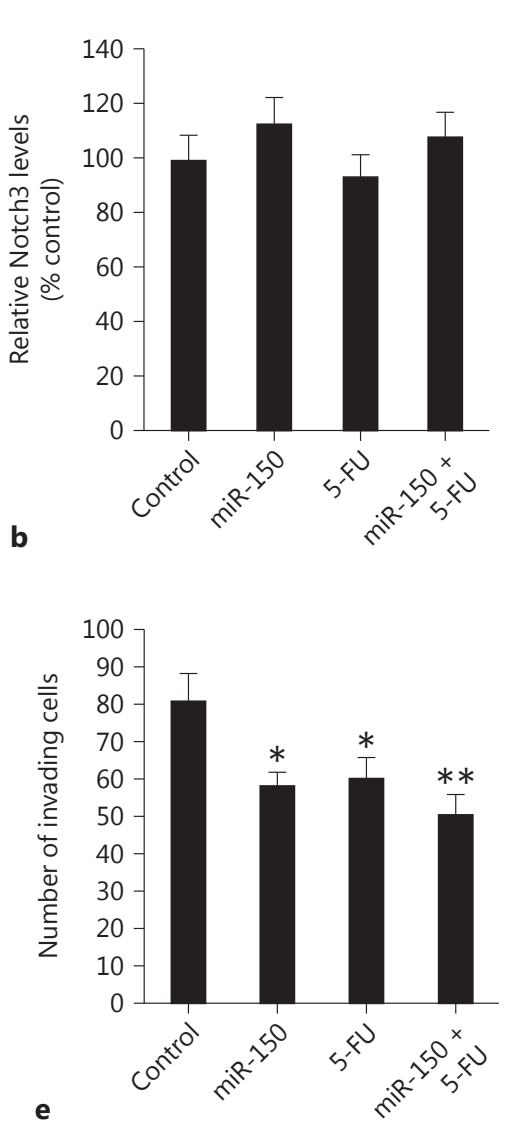
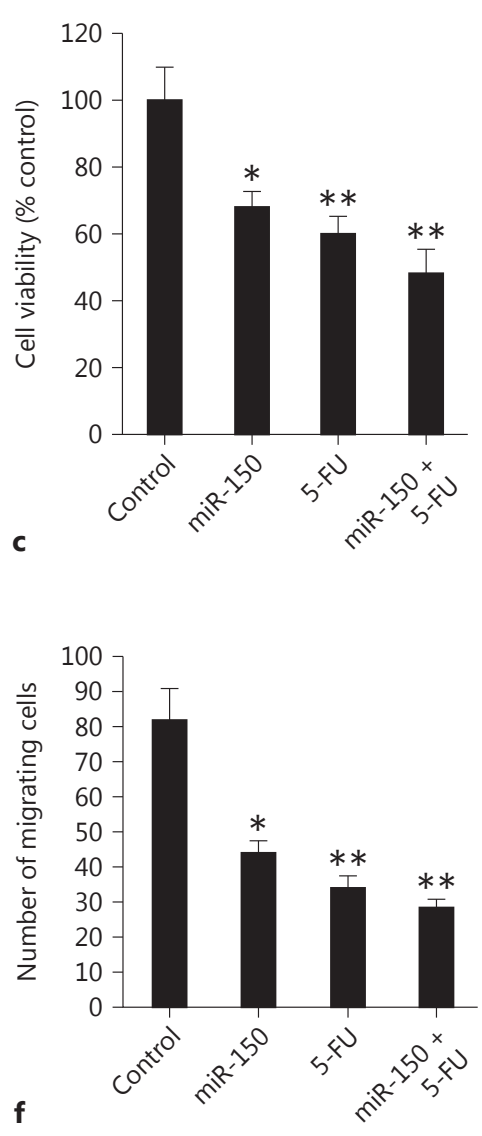

Fig. 5. The antitumor effect of miR-150 in BxPC-3 cells. Cells were transfected with miR-150 mimic, 5-FU incubation or combined miR-150 mimic and 5-FU incubation. a Representative Western blots of Notch 3 and $\alpha$-tubulin protein. $\mathbf{b}$ Semiquantitative analysis of Notch3 receptor protein expression relative to controls (100\%). $\mathrm{n}=5$. c Cell viability was analyzed by MTT. $\mathbf{d}$ Percentage of apoptotic cells in Hoechst 33342 staining. e Cell invasion analysis. f Cell migration analysis. ${ }^{*} \mathrm{p}<0.01,{ }^{* *} \mathrm{p}<0.001$ versus controls. $\mathrm{n}=5$. ered in the plasma of PDAC patients and in PDAC tumor tissues. However, a lower plasma level of miR-34a predicted poor prognosis and significantly correlated with larger tumor size, high TNM stage, invasion and lymph node metastasis in PDAC patients. In contrast, although miR-150 significantly correlated with high TNM stage, it had no significant associations with tumor size, invasion, lymph node metastasis or survival. Importantly, miR$34 \mathrm{a}$, but not miR-150, was involved in the chemoresistance of PDAC cells to 5-FU. Further molecular studies revealed that miR-34a was involved in the chemoresistance and the malignant phenotypes of PDAC cells, possibly through regulating the expression of Notch1, Notch2, and Notch4 receptors (but not the Notch3 receptor).
Currently, only a few chemotherapeutic agents, including 5-FU, have consistently shown some degree of beneficial effects in advanced pancreatic cancer [18]. Chemoresistance is one of the main obstacles that limit the therapeutic effects of drugs, but the mechanisms that contribute to this have not been fully elucidated. Consistent with previous findings $[19,20]$, we found that miR$34 \mathrm{a}$ influences the response of PDAC cells to 5-FU. Our study suggests that the loss of miR-34a expression may be associated with the chemoresistance of PDAC tumor cells. In contrast, although miR-150 mimic significantly inhibited the malignant behavior of PDAC cells, the combination of miR-150 mimic and 5-FU treatment exhibited no significant effect in sensitizing the anticancer effect of 5-FU. These findings suggest that a loss of miR- 
150 expression may not be associated with chemoresistance in PDAC.

This study further explored the molecular and cellular mechanisms involved in the chemoresistance of PDAC tumor cells to 5 -FU. The studies in vivo in xenograft tumor tissues and in vitro in PDAC cells revealed that miR34a mimic significantly inhibited CD133, Notch1, Notch2 and Notch4, but not Notch3, protein expression. Moreover, the combination of miR-34a mimic and 5-FU treatment was more effective in inhibiting the malignant behavior of PDAC cells in vitro and in vivo. CD133 is a cancer stem cell marker and cancer stem cells are widely thought to participate in the chemoresistance of a variety of cancer cells [21,22]. Notch signaling has been demonstrated to regulate cancer stem cell survival [23, 24]. Therefore, our findings suggest that miR-34a may exert antichemoresistant effects through inhibiting Notch signaling in cancer stem cells. However, we acknowledge that miR-34a may target many genes. Notch signaling may not be the only signaling regulated by miR-34a. Conversely, the miR-150 mimic showed no effect on Notch3 expression. Srivastava et al. [25] demonstrated that miR150 directly targets MUC4 and suppresses the malignant behavior of pancreatic cancer cells. Farhana et al. [26] showed that the overexpression of miR-150 diminishes c-Myb and Bcl-2 protein levels. Thus, miR-150 may exert an anticancer effect through regulating other signaling pathways, but not Notch signaling.
A previous study proposed that miR-34a is a potentially useful marker for the diagnosis of PDAC [8]. Our study demonstrated that lower miR-34a levels correlate with severe clinical symptoms and poor prognosis in PDAC patients. Thus, miR-34a is a tumor suppressor in $\mathrm{PDAC}$ and the loss of miR-34a expression is a biomarker for poor prognosis of PDAC patients. miR-34a can also be used as a marker for the diagnosis of PDAC or a target for developing targeting therapy. This study first demonstrated that miR-150 expression was also significantly decreased in the serum and tumor tissues of PDAC patients. Although the lower miR-150 expression correlated with the higher clinical stage, it only showed a tendency to be associated with poor prognosis $(\mathrm{p}=0.053)$. Therefore, miR-150 is not an ideal marker or target for PDAC.

In conclusion, miR-34a functions as a tumor suppressor and the loss of miR-34a expression is a biomarker for clinical severity and poor prognosis in PDAC. miR-34a is also involved in the malignant behavior and chemoresistance of pancreatic cancer, possibly through regulating Notch signaling in cancer stem cells. In contrast, miR-150 is not an ideal marker for the progression and prognosis of PDAC.

\section{Disclosure Statement}

The authors declare no conflicts of interest.

\section{References}

1 Jemal A, Siegel R, Ward E, et al: Cancer statistics, 2009. CA Cancer J Clin 2009;59:225-249.

-2 Siegel R, Ma J, Zou Z, et al: Cancer statistics, 2014. CA Cancer J Clin 2014;64:9-29.

$\checkmark 3$ Teague A, Lim KH, Wang-Gillam A: Advanced pancreatic adenocarcinoma: a review of current treatment strategies and developing therapies. Ther Adv Med Oncol 2015;7: 68-84.

4 Puleo F, Maréchal R, Demetter P, et al: New challenges in perioperative management of pancreatic cancer. World J Gastroenterol 2015;21:2281-2293.

5 Cai Y, Yu X, Hu S, et al: A brief review on the mechanisms of miRNA regulation. Genomics Proteomics Bioinformatics 2009;7:147-154.

6 Chitkara D, Mittal A, Mahato RI: miRNAs in pancreatic cancer: therapeutic potential, delivery challenges and strategies. Adv Drug Deliv Rev 2015;81:34-52.
Jamieson NB, Morran DC, Morton JP, et al: MicroRNA molecular profiles associated with diagnosis, clinicopathologic criteria, and overall survival in patients with resectable pancreatic ductal adenocarcinoma. Clin Cancer Res 2012;18:534-545.

-8 Alemar B, Izetti P, Gregório C, et al: miRNA21 and miRNA-34a are potential minimally invasive biomarkers for the diagnosis of pancreatic ductal adenocarcinoma. Pancreas 2016;45:84-92.

-9 Ikeda Y, Tanji E, Makino N, Kawata S, Furukawa T: MicroRNAs associated with mitogenactivated protein kinase in human pancreatic cancer. Mol Cancer Res 2012;10:259-269.

10 Ji Q, Hao X, Zhang M, et al: MicroRNA miR34 inhibits human pancreatic cancer tumorinitiating cells. PLoS One 2009;4:e6816.

11 Xia J, Duan Q, Ahmad A, et al: Genistein inhibits cell growth and induces apoptosis through up-regulation of miR-34a in pancreatic cancer cells. Curr Drug Targets 2012;13:1750-1756.
12 Ghisi M, Corradin A, Basso K, et al: Modulation of microRNA expression in human Tcell development: targeting of NOTCH3 by miR-150. Blood 2011;117:7053-7062.

13 Srivastava SK, Bhardwaj A, Singh S, et al: MicroRNA-150 directly targets MUC4 and suppresses growth and malignant behavior of pancreatic cancer cells. Carcinogenesis 2011; 32:1832-1839.

14 Zhang Y, Zhu X, Bai M, et al: Maternal deprivation enhances behavioral vulnerability to stress associated with miR-504 expression in nucleus accumbens of rats. PLoS One 2013; 8:e69934.

15 Filgueiras Mde C, Morrot A, Soares PM, et al: Effects of 5-fluorouracil in nuclear and cellular morphology, proliferation, cell cycle, apoptosis, cytoskeletal and caveolar distribution in primary cultures of smooth muscle cells. PLoS One 2013;8:e63177.
miR-34a in PDAC and Associated

Molecular and Cellular Mechanisms
Pathobiology 2016;84:38-48 DOI: $10.1159 / 000447302$ 
16 Zhang X, Kon T, Wang H, et al: Enhancement of hypoxia-induced tumor cell death in vitro and radiation therapy in vivo by use of small interfering RNA targeted to hypoxia-inducible factor-1alpha. Cancer Res 2004;64:81398142.

17 Saito Y, Nakaoka T, Saito H: MicroRNA-34a as a therapeutic agent against human cancer. J Clin Med 2015;4:1951-1959.

18 Neoptolemos JP, Cunningham D, Friess H, et al: Adjuvant therapy in pancreatic cancer: historical and current perspectives. Ann Oncol 2003; 14:675-692.

19 Garajová I, Le Large TY, Frampton AE, et al: Molecular mechanisms underlying the role of microRNAs in the chemoresistance of pancreatic cancer. Biomed Res Int 2014;2014: 678401.
20 Frampton AE, Krell J, Jamieson NB, et al: MicroRNAs with prognostic significance in pancreatic ductal adenocarcinoma: a meta-analysis. Eur J Cancer 2015;51:1389-1404.

21 Matsuda Y, Kure S, Ishiwata T: Nestin and other putative cancer stem cell markers in pancreatic cancer. Med Mol Morphol 2012; 45:59-65.

22 Zhou Q, Chen A, Song H, et al: Prognostic value of cancer stem cell marker CD133 in ovarian cancer: a meta-analysis. Int J Clin Exp Med 2015;8:3080-3088.
23 An SM, Ding Q, Zhang J, et al: Targeting stem cell signaling pathways for drug discovery: advances in the Notch and Wnt pathways. Sci China Life Sci 2014;57:575-580.

24 Lobry C, Oh P, Mansour MR, et al: Notch signaling: switching an oncogene to a tumor suppressor. Blood 2014;123:2451-2459.

25 Srivastava SK, Bhardwaj A, Singh S, et al: MicroRNA-150 directly targets MUC4 and suppresses growth and malignant behavior of pancreatic cancer cells. Carcinogenesis 2011; 32:1832-1839.

26 Farhana L, Dawson MI, Murshed F, Das JK, Rishi AK, Fontana JA: Upregulation of miR150 and miR-630 induces apoptosis in pancreatic cancer cells by targeting IGF-1R. PLoS One 2013;8:e61015. 